\title{
Dynamic changes in the level of WT1 as an MRD marker to predict the therapeutic outcome of patients with AML with and without allogeneic stem cell transplantation
}

\author{
HUASHENG LIU, XIAONING WANG, HAILING ZHANG, JINCHENG WANG, YING CHEN, \\ TIANTIAN MA, JING SHI, YA KANG, JIEYING XI, MENGCHANG WANG and MEI ZHANG \\ Department of Hematology, First Affiliated Hospital of Xi'an Jiaotong University, Xi'an, Shaanxi 710061, P.R. China
}

Received July 4, 2018; Accepted May 14, 2019

DOI: $10.3892 / \mathrm{mmr} .2019 .10440$

\begin{abstract}
Monitoring minimal residue disease (MRD) is an effective approach to evaluate the response to chemotherapy, and it is used to select the ideal therapeutic strategy and to predict the recurrence during therapy for hematological disorders. The Wilm's tumor 1 (WT1) gene, which is highly expressed in $>80 \%$ of patients with acute myeloid leukemia (AML) and its increased expression level may cause poor clinical outcomes, is a potential MRD marker of hematological neoplasms. In the present study, the expression levels of WT1 and other molecular markers were retrospectively analyzed by reverse transcription-quantitative PCR in 195 patients with AML. The expression level of WT1 was significantly lower in patients with remission compared with patients with early-stage and recurrent AML. Moreover, WT1 expression was significantly decreased in patients with RUNX family transcription factor 1-RUNX1 translocation partner 1 fusion, but higher in patients with promyelocytic leukemia-retinoic acid receptor $\alpha$ fusion. WT1 expression was significantly reduced during remission. In patients with AML who underwent allogeneic hematopoietic stem cell transplantation (allo-HSCT), the mortality rate 2 years after allo-HSCT was significantly lower in patients with low expression level of WT1 compared with subjects presenting high expression level of WT1. Collectively, the upregulation of the expression level of WT1 in combination with the identification of other genetic abnormalities may be used as MRD markers of hematological neoplasms.
\end{abstract}

Correspondence to: Dr Huasheng Liu or Dr Mei Zhang, Department of Hematology, First Affiliated Hospital of Xi'an Jiaotong University, 277 West Yanta Road, Xi'an, Shaanxi 710061, P.R. China

E-mail: lhs681995@126.com

E-mail: zhangmei@medmail.com.cn

Key words: acute myeloid leukemia, Wilm's tumor 1, reverse transcription-quantitative PCR, minimal residue disease

\section{Introduction}

Acute myeloid leukemia (AML) is a hematological disorder that results from the abnormal differentiation and proliferation of hematopoietic cells, and chemotherapy represents the standard therapeutic treatment for patients with $\operatorname{AML}(1,2)$. Despite the high response rate to chemotherapy, relapse frequently occurs after remission, lowering the overall response rate in patients with AML, making post-remission therapy necessary $(3,4)$. Therefore, it is necessary to find minimal residual disease (MRD) markers in order to evaluate the curative effects of therapeutic treatments and predict relapse of AML. At present, to the best of our knowledge, the main techniques for detecting MRD are multi-parameter flow cytometry (MFC) and reverse transcription-quantitative (RT-q)PCR $(5,6)$. However, due to limitations in detecting the full spectrum of relapse risks, MFC is not an ideal methodology for MRD monitoring. Accumulating evidence indicated that RT-qPCR, which is used for quantitative determination of fusion genes, genetic mutations and overexpression of oncogenes, is more sensitive than MFC in MRD monitoring $(7,8)$. Despite the high sensitivity and accuracy of RT-qPCR, detection of biomarkers is only suitable for patients with certain fusion genes, such as RUNX family transcription factor 1 (RUNX1)-RUNX1 translocation partner 1 (ETO) $(9,10)$. Therefore, it is required to identify additional molecular markers of MRD suitable for all patients.

Wilm's tumor 1 (WT1) gene, encoding a zinc-finger transcription factor, was originally identified as a tumor suppressor gene regulating hematopoiesis and apoptosis (11). Previous studies have revealed that WT1 is highly expressed in $>80 \%$ of patients with AML and may cause poor clinical outcomes due to an increased resistance to apoptosis (12-15). Despite these previous studies, to the best of our knowledge, WT1 has not been used as an established marker for MRD monitoring in hematological malignancies. Frairia et al (15) observed that WT1 is a marker of recurrence after complete remission and prior to allogeneic hematopoietic stem cell transplantation (allo-HSCT), and patients with high WT1 expression had a significantly higher 2-year cumulative incidence of relapse compared to those with low WT1 levels. Therefore, low and high expression of WT1 may be associated with clinical remission and relapse, respectively, and WT1 may be a potential 
prognostic factor and a therapeutic target in patients with AML. By contrast, other previous studies observed that WT1 may not be used in MRD detection (16) or that WT1 upregulation may be associated with favorable outcomes in patients with AML (17).

In the present study, to clarify these discrepancies, the expression levels of WT1 and other molecular markers, such as genetic mutations, were retrospectively analyzed in 195 patients with AML by RT-qPCR. Additionally, the association between the expression levels of WT1 and various genetic markers were investigated, and the prognostic value of WT1 expression in patients with AML after allo-HSCT was examined.

\section{Materials and methods}

Patient samples. A total of 195 patients with AML were enrolled in the First Affiliated Hospital of Xi'an Jiaotong University. Written informed consent was obtained from all patients. The present retrospective study was approved by The Institutional Ethics Committee of The First Affiliated Hospital of Xi'an Jiaotong University. Diagnoses were performed according to the French-American-British diagnostic criteria (FAB) (18) for AML and combined with immunophenotyping, cytogenetics and molecular biological assays, as previously described (19). The 195 patients were diagnosed with AML and received therapy between January 2013 and September 2017. The cohort of patients included 102 men and 93 women; the median age was 45 years, ranging between 7 and 76 years. In total, 169 patients received chemotherapy and 31 received allo-HSCT. Based on FAB criteria, 2, 7, 100, 21, 32, 18 and 3 patients were categorized as M0, M1, M2, M3, M4, M5 and M6, respectively.

Identification of AML related fusion genes and mutations. Total RNA was extracted from bone marrow using TRIzol reagent (Invitrogen; Thermo Fisher Scientific, Inc.) following the manufacturer's protocol. Extracted RNA samples were treated with DNase I (Applied Biosystems; Thermo Fisher Scientific, Inc.) to remove any DNA contamination before one-step RT-qPCR. These RNA samples were then subjected to one-step RT-qPCR to detect AML-related fusion transcripts using The Leukemia Related Fusion gene detection kit (Shanghai Yuanqi Bio-Pharmaceutical Co., Ltd.) according to the manufacturer's protocol. Analysis of positive and negative controls was performed according to the manufacturer's protocol. The primers were included in the kit. The mixture of each reaction contained $15 \mu$ l total RNA, $8 \mu$ l RT-PCR Buffer, $2 \mu 1$ Multiplex Enzyme Mix (Shanghai Yuanqi Bio-Pharmaceutical Co., Ltd.) in a total volume of $25 \mu \mathrm{l}$. The Taqman RT-qPCR reaction was performed at $42^{\circ} \mathrm{C}$ for $30 \mathrm{~min}$, at $94^{\circ} \mathrm{C}$ for $5 \mathrm{~min}$ followed by 40 cycles of $94^{\circ} \mathrm{C}$ for $15 \mathrm{sec}$ and $60^{\circ} \mathrm{C}$ for $1 \mathrm{~min}$ on the 7300 Real Time PCR System (Thermo Fisher Scientific, Inc.). Sanger sequencing was conducted to detect AML-related mutations. DNA was extracted from bone marrow with DNeasy Blood \& Tissue Kit (Qiagen, Inc.) for Sanger sequencing by Leukemia Related Gene Test Kit (Shanghai Yuanqi Bio-Pharmaceutical Co., Ltd.). The primers were included in the kit. PCR reactions were carried out in a final volume of $25 \mu \mathrm{l}$ containing $3 \mu \mathrm{l}$ genomic DNA, $9 \mu \mathrm{l}$ sequencing reaction, and $13 \mu \mathrm{l}$ PCR MIX3 (Shanghai Yuanqi Bio-Pharmaceutical Co., Ltd.). Samples were processed at $42^{\circ} \mathrm{C}$ for $5 \mathrm{~min}$ and at $94^{\circ} \mathrm{C}$ for $5 \mathrm{~min}$, followed by 40 cycles at $94^{\circ} \mathrm{C}$ for $30 \mathrm{sec}, 58^{\circ} \mathrm{C}$ for $30 \mathrm{sec}$, and $72^{\circ} \mathrm{C}$ for $60 \mathrm{sec}$, with a final step for $5 \mathrm{~min}$ at $72^{\circ} \mathrm{C}$. PCR products were loaded on agarose gels, purified and sequenced using BigDye Terminators and ABI 3500 Genetic Analyzer (Thermo Fisher Scientific, Inc.).

Quantitative expression of WT1. In total, $2 \mathrm{ml}$ bone marrow (BM) was extracted from the patients. Total RNA from $\mathrm{BM}$ samples was isolated using TRIzol reagent (Invitrogen; Thermo Fisher Scientific, Inc.) according to the manufacturer's protocol. RNA was treated with Amplification Grade DNase I (Takara Bio, Inc.) at room temperature for $60 \mathrm{~min}$ to prevent DNA contamination. A total of $5 \mu 1 \mathrm{RNA}(\sim 500 \mathrm{ng})$ was used to detect WT1 expression using a one-step RT-qPCR WT1 kit (Shanghai Yuanqi Bio-Pharmaceutical Co., Ltd.) according to the manufacturer's protocol. According to the manufacturer's protocol, the house-keeping Abelson gene (ABL) was used as the internal control to evaluate the relative levels of WT1 expression, as previously described $(20,21)$. The primers were included in the RT-qPCR WT1 kit (Shanghai Yuanqi Bio-Pharmaceutical Co., Ltd.). The Taqman PCR mixtures were prepared according to the manufacturer's protocol and the reactions were performed using an ABI 7500 real-time PCR instrument (Thermo Fisher Scientific, Inc.). The thermocycling conditions were as follows: Initial incubations at $42^{\circ} \mathrm{C}$ for $30 \mathrm{~min}$ and $94^{\circ} \mathrm{C}$ for $5 \mathrm{~min}$, followed by 40 cycles of $94^{\circ} \mathrm{C}$ for $15 \mathrm{sec}$ and $60^{\circ} \mathrm{C}$ for $60 \mathrm{sec} 40$ cycles. All experiments were repeated three times with appropriate positive and negative controls. WT1 levels were expressed as the number of WT1 copies per 100 copies of ABL. Relative quantification was performed using standard reference curves according to the manufacturer's protocol as previously described (22-24). The detection threshold of WT1 was $0.02 \%$ of ABL gene copies, as previously described $(20,21)$.

Statistical analysis. All experiments were repeated three times. All data were analyzed using SPSS software (version 20.0; IBM Corp.). Data are presented as the mean \pm SEM. Differences of WT1 expression between two groups were analyzed using Student's t-test. Differences among multiple groups were determined by one-way or two-way ANOVA followed by Tukey's post hoc test. Repeated measures ANOVA was performed for the analysis of dependent variables. The effects of allo-HSCT on overall survival (OS) rates were analyzed using Kaplan-Meier curves. $\mathrm{P}<0.05$ was considered to indicate a statistically significant difference.

\section{Results}

WT1 expression in AML. The expression level of WT1 in BM samples from patients with AML was assessed by RT-qPCR analysis. The expression level of WT1 at initial diagnosis was significantly higher in patients with no response (NR) than in those with complete response (CR) (Fig. 1). In line with a previous study (25), CR was characterized by: i) Bone marrow blasts $<5 \%$; ii) the absence of blasts with Auer rods; iii) the absence of extramedullary disease; iv) an absolute neutrophil count $>1.0 \times 10^{9} / 1$; and v) a platelet count $>100 \times 10^{9} / 1$. Partial 
Table I. WT1 expression in AML subtypes at diagnosis.

\begin{tabular}{lrc}
\hline Subtype & $\mathrm{n}$ & Relative WT1 expression, \% \\
\hline M1 & 2 & $2.77 \pm 1.272$ \\
M2 & 35 & $8.44 \pm 1.596$ \\
M3 & 10 & $48.30 \pm 22.43$ \\
M4 & 12 & $52.39 \pm 20.74$ \\
M5 & 8 & $5.97 \pm 2.189$ \\
M6 & 3 & $34.22 \pm 11.18$
\end{tabular}

Relative expression level of WT1 was calculated as percentage of the expression level of ABL. WT1, Wilm's Tumor 1; ABL, Abelson gene.

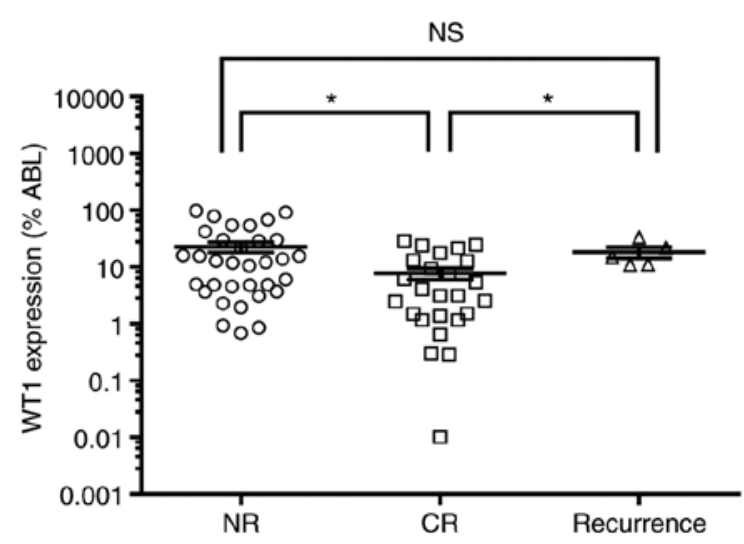

Figure 1. Initial expression level of WT1 in bone marrow samples from patients with acute myeloid leukemia. Patients who exhibited NR, CR without recurrence and CR with recurrence were analyzed. Data are presented as the mean \pm SEM. ${ }^{*} \mathrm{P}<0.05$. WT1, Wilm's Tumor 1 ; NS, not significant; NR, no reponse; $\mathrm{CR}$, complete response, $\mathrm{ABL}$, Abelson gene.

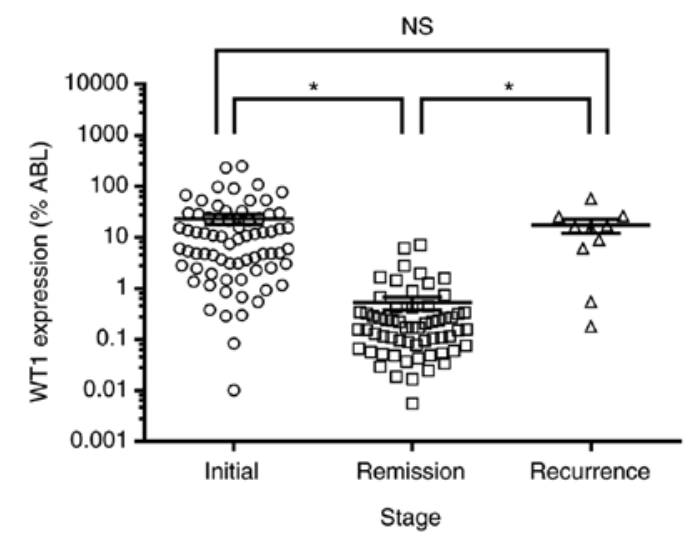

Figure 2. WT1 expression in patients with AML who did not receive allogeneic hematopoietic stem cell transplantation. Reverse transcription-quantitative PCR analysis was performed to assess WT1 expression in patients with AML at initial diagnosis, remission and recurrence stage. Data are presented as the mean \pm SEM. ${ }^{*} \mathrm{P}<0.05$. WT1, Wilm's Tumor 1 ; AML, acute myeloid leukemia; NS, not significant; ABL, Abelson gene.

remission (PR) was defined as $5-20 \%$ bone marrow blasts and a $50 \%$ decrease in bone marrow blasts during pretreatment. Patients who did not exhibit CR or PR after chemotherapy were categorized in the NR group. Moreover, after diagnosis, the expression level of WT1 in patients with recurrence was significantly higher than in CR patients, but not significantly different than in NR patients (Fig. 1). The expression level of WT1 was assessed in patients with AML at different stages of the disease: i) Initial; ii) remission; and iii) recurrence. The present RT-qPCR results suggested that WT1 expression was significantly lower in patients with remission than in those with initial and recurrent AML (Fig. 2). The expression level of WT1 was not significantly different between patients with initial and recurrent AML. Furthermore, the expression of WT1 was analyzed in patients with different AML subtypes according to the FAB criteria. The expression level of WT1, calculated as a percentage of ABL expression, was highest in the M3 (48.3\%) and M4 (52.4\%) subtypes and lowest in the M1 subtype (2.8\%). Patients in the subtypes M6, M2 and M5 exhibited a relative expression level of WT1 of 34.2, 8.4 and $6.0 \%$, respectively (Table I). There were no significant differences in ABL expression levels among different subtypes (data not shown). Importantly, further studies are required to evaluate the expression level of WT1 in a higher number of patients with various subtypes of AML.

Association between WT1 expression and genetic mutations. The present study investigated the association between the expression level of WT1 and the presence of fusion genes, including RUNX1-ETO, breakpoint cluster region-ABL, core-binding factor subunit $\beta$-myosin heavy chain 11 and promyelocytic leukemia (PML)-retinoic acid receptor $\alpha$ (RARA). In addition, the present study investigated the association between the expression level of WT1 and prognosis-associated mutations affecting genes such as CCAAT enhancer binding protein $\alpha$ (CEBPA), mast/stem cell growth factor receptor, FMS-like tyrosine kinase-3 internal tandem duplication (FLT3-ITD), nucleophosmin 1 and DNA methyltransferase $3 \alpha$. All patients positive for WT1 expression presented fusion genes or genetic mutations; however, 97.4\% of patients with RUNX1-ETO mutations were positive for WT1 expression, and $94.7 \%$ of patients with FLT3-ITD exhibited a positive expression of WT1. In addition, the association between the expression level of WT1 and the aforementioned genetic anomalies was investigated. Compared with patients without genetic anomalies, WT1 expression was significantly lower in patients presenting the gene fusion RUNX1-ETO, but was not significantly altered in patients with other mutations or fusion genes. However, WT1 expression was higher in patients with PML-RARA than in those with RUNX1-ETO or CEBPA (Table II). In addition, the level of WT1 expression was significantly reduced after molecular remission (26) in patients exhibiting RUNX1-ETO and PML-RARA mutations at initial diagnosis (Fig. 3).

WT1 upregulation is associated with higher mortality rates in patients with AML after allo-HSCT. Among the 195 patients with AML, 31 patients received allo-HSCT. Following allo-HSCT treatment, WT1 expression decreased significantly after 1, 3 and 6 months compared with before allo-HSCT treatment (Fig. 4A). The patients who underwent allo-HSCT were divided two groups: i) High expression level of WT1; and ii) low expression level of WT1. The mean relative expression level (3.42\% compared to ABL) before allo-HSCT was 
Table II. Comparison between WT1 expression and various genetic mutations.

\begin{tabular}{lrcc}
\hline $\begin{array}{l}\text { Genetic } \\
\text { mutation }\end{array}$ & $\mathrm{n}$ & $\begin{array}{l}\text { Relative WT1 } \\
\text { expression, } \%\end{array}$ & P-value \\
\hline No mutations & 12 & $17.27 \pm 4.63$ & - \\
RUNX1-ETO & 14 & $6.81 \pm 2.23$ & $0.0435^{\mathrm{a}}$ \\
PML-RARA & 9 & $52.22 \pm 24.69$ & 0.1266 \\
CEBPA & 12 & $13.73 \pm 4.47$ & 0.5877 \\
FLT3-ITD & 12 & $31.85 \pm 18.95$ & 0.4629
\end{tabular}

Relative expression level of WT1 was calculated as percentage of the expression level of Abelson gene. ${ }^{a} \mathrm{P}<0.05$ vs. No mutations. WT1, Wilm's Tumor 1; RUNX1-ETO, runt related transcription factor 1-RUNX1 translocation partner 1; PML-RARA, promyelocytic leukemia-retinoic acid receptor $\alpha$; CEBPA, CCAAT enhancer binding protein $\alpha$; FLT3-ITD, FMS-like tyrosine kinase-3 internal tandem duplication.

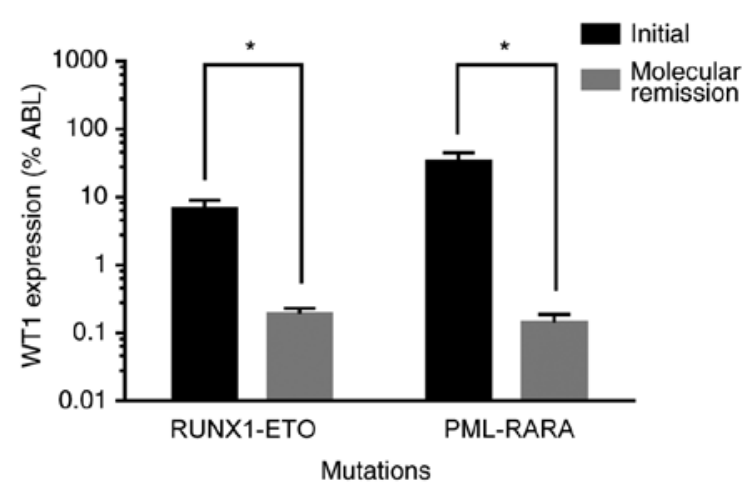

Figure 3. WT1 expression correlates with fusion gene changes at remission. Reverse transcription-quantitative PCR was used to assess WT1 expression in patients positive for RUNX1-ETO and PML-RARA fusion genes before and after remission. ${ }^{*} \mathrm{P}<0.05$. WT1, Wilm's Tumor 1 ; RUNX1, runt related transcription factor 1; ETO, RUNX1 translocation partner 1; PML, promyelocytic leukemia; RARA, retinoic acid receptor $\alpha$; ABL, Abelson gene.

selected as threshold to divide the two groups. The 2-year OS after allo-HSCT in the WT1 low expression group was $93.3 \%$ $(14 / 15)$, whereas in the WT1 high expression group was $\sim 60 \%$ (10/16; Fig. 4B). Compared with the expression level of WT1 before allo-HSCT, WT1 expression after allo-HSCT was not significantly altered in patients deceased within 2 years after allo-HSCT. By contrast, the expression level of WT1 decreased to $<10 \%$ in surviving patients (Fig. 4C). The present results suggested that the dynamic changes in the expression levels of WT1 could be used to predict the treatment outcome and disease state (Fig. 5). WT1 expression decreased significantly and was maintained at low levels during the remission stage (Fig. 5A), but increased at recurrence stage (Fig. 5B). Following allo-HSCT, patients with AML at remission stage exhibited low levels of WT1 (Fig. 5C), and WT1 expression level was significantly increased at recurrence stage or prior to mortality (Fig. 5D). The present results suggested that the expression level of WT1 was negatively associated with the therapeutic response in patients with AML who underwent allo-HSCT.

\section{Discussion}

Monitoring MRD has become one of the most effective approaches to determine prognosis and therapeutic strategies in patients with AML. WT1 serves an important role in blast cell survival by enhancing proliferation and inhibiting apoptosis, and WT1 is upregulated in the majority of patients with initial AML (27). However, the prognostic potential of the expression level of WT1 remains unclear. Here, we retrospectively investigated the prognostic potential of WT1 in a cohort of patients with AML.

WT1 expression level is higher in patients with AML at diagnosis compared with healthy patients, decreases at complete remission, and increases prior to clinical relapse $(28,29)$. In the present study, the expression levels of WT1 in patients with AML in initial, remission and recurrence stage are in line with a previous study indicating that low and high expression of WT1 in AML are associated with clinical remission and relapse, respectively (20). Therefore, the present results suggested that recurrence can be predicted based on the expression level of WT1 in patients with AML, in line with the previous study by Mashima et al (30). In addition, WT1 expression is high in $>80 \%$ of patients with AML and expression of WT1 decreases when patients entered remission (31). Therefore, WT1 expression could potentially be used for MRD detection in patients with AML, in particular in patients with no cytogenetic or molecular abnormalities.

Hao et al (32) showed that WT1 expression was highest in the M3 subtype and lowest in the M1 subtype. In the present study, lowest WT1 expression was detected in the M1 subtype. However, high levels of WT1 were observed in both the M3 and M4 subtypes. In addition, the expression level of WT1 was identified to be associated with genetic abnormalities and the average WT1 expression was higher in patients with CEBPA mutations compared with patients exhibiting no mutations in the CEBPA gene. In addition, the expression level of WT1 was increased in patients with initial AML presenting FLT3-ITD mutation. The present results are in line with the study by Lyu et al (14), suggesting that patients with FLT3-ITD mutation presented high levels of WT1 compared with other patients with wild-type FLT3. Furthermore, compared with patients without identified mutations, WT1 expression was lower in patients with RUNX1-ETO and higher in patients with PML-RARA. The present results suggested that the expression level of WT1 was associated with the outcome of patients with AML. In addition, the level of WT1 expression was significantly reduced after molecular remission in patients exhibiting RUNX1-ETO and PML-RARA mutations at initial diagnosis, suggesting that WT1 could be used as an MRD marker in the majority of patients with AML without specific fusion genes.

Duléry et al (33) reported that the 3-year event-free survival rate is reduced in WT1-based MRD positive patients compared with WT1-based MRD negative patients, and patients who underwent allo-HSCT positive for WT1 expression after 3 months exhibited an unfavorable prognosis, suggesting a detrimental role for WT1 in relapse. In the present study, although no patients exhibited recurrence following allo-HSCT, the mortality rate 2 years after allo-HSCT was higher for patients in the high WT1 expression 

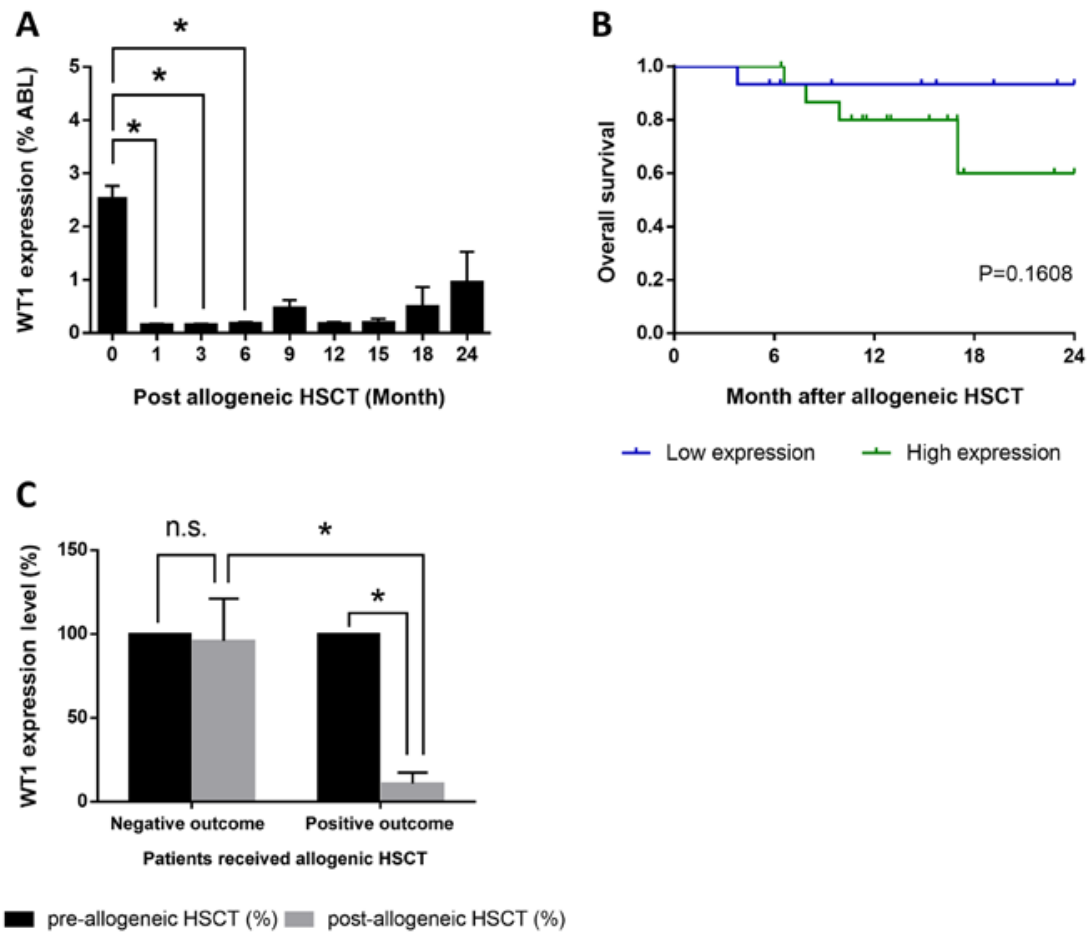

- Low expression - High expression

Figure 4. WT1 expression in patients with AML. (A) WT1 expression was assessed by reverse transcription-quantitative PCR at various time points following allo-HSCT. (B) Kaplan-Meier curve of 2-year overall survival rates in different WT1 expression groups following allo-HSCT. (C) Comparison of WT1 expression levels before and after allo-HSCT in patients with AML. Data are presented as the mean \pm SEM. ${ }^{*} \mathrm{P}<0.05$. WT1, Wilm's Tumor 1 ; allo-HSCT, allogeneic hematopoietic stem cell transplantation; NS, not significant; ABL, Abelson gene; AML, acute myeloid leukemia.
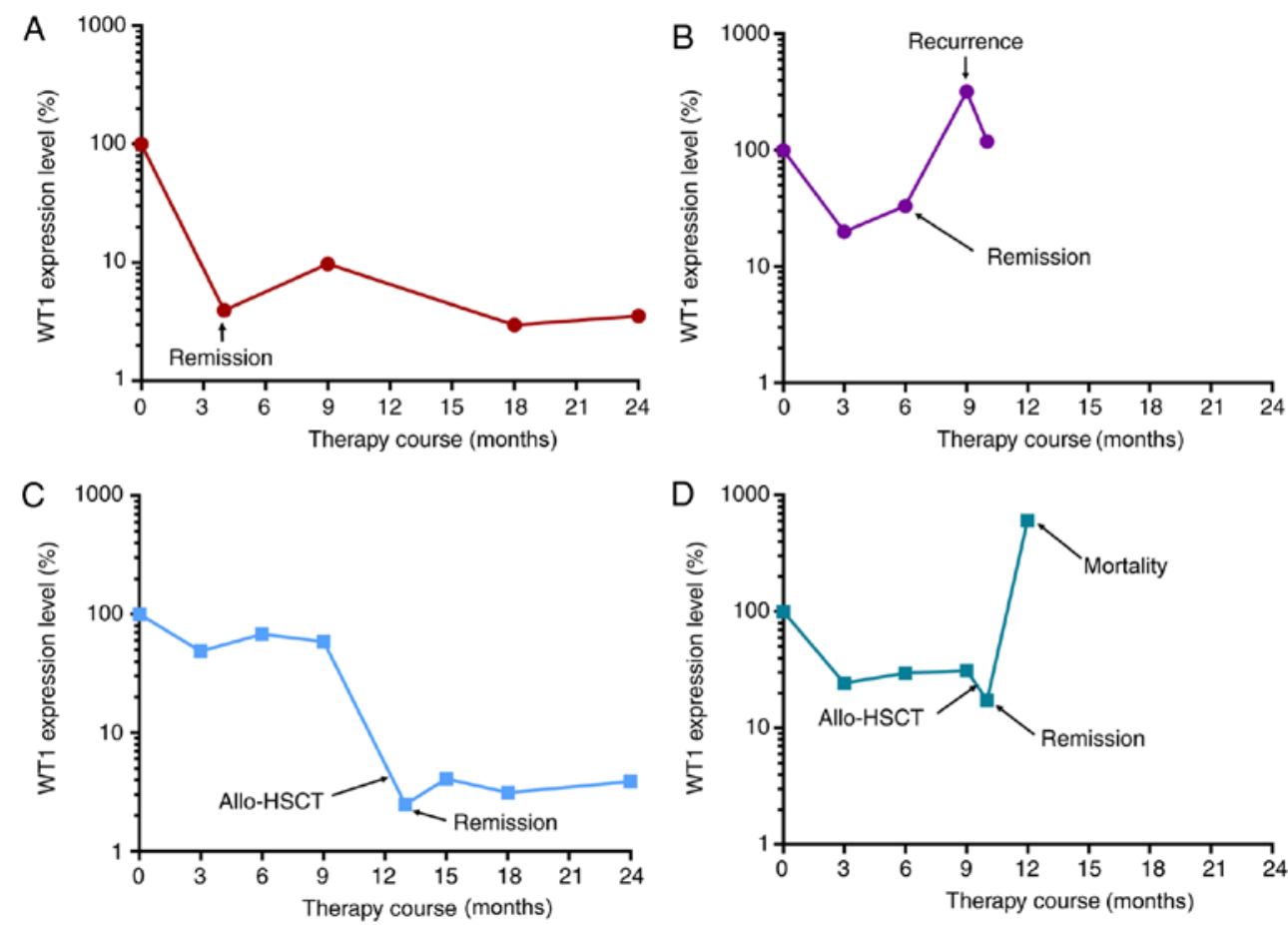

Figure 5. WT1 levels in patients with acute myeloid leukemia. Patients exhibiting (A) remission and (B) recurrence. Patients who received allo-HSCT presenting (C) remission and (D) mortality. WT1, Wilm's Tumor 1; allo-HSCT, allogeneic hematopoietic stem cell transplantation.

group compared with those with low expression of WT1. The present results suggested that WT1 expression was negatively associated with therapeutic effect of allo-HSCT in patients with AML. The present results are in line with a previous study by Candoni et al (34) that showed a better outcome for
WT1-negative patients compared with WT1-positive patients. Collectively, the present study identified that WT1 expression may be associated with the prognosis of patients with AML, including those that received allo-HSCT. Therefore, WT1 could be considered as an MRD biomarker for AML. Due to 
the limitations of the present study, including the inconsistent number of patients with different AML subtypes, further studies analyzing a higher number of patients with AML are required in order to validate the present results.

\section{Acknowledgements}

Not applicable.

\section{Funding}

The present study was supported by grants from The Shaanxi Province Science and Technology Research Projects (grant no. 2016SF-122) and the Clinic Research Award of the First Affiliated Hospital of Xi'an Jiaotong University (grant no. XJTU1AHCR2014-032).

\section{Availability of data and materials}

The datasets used and/or analyzed during the current study are available from the corresponding author on reasonable request.

\section{Authors' contributions}

HL collected the data, wrote and revised the present manuscript. XW and MZ assisted in writing the manuscript. HZ and JW performed the RT-qPCR experiments. XW, TM, JS and MZ performed statistical and data analyses. YC, YK, JX and MW managed the patient information and contributed to data acquisition. All authors read and approved the final manuscript.

\section{Ethics approval and consent to participate}

The present study was approved by The Institutional Ethics Committee of The First Affiliated Hospital of Xi'an Jiaotong University. Informed consent was obtained from all patients.

\section{Patient consent for publication}

Informed consent was obtained from all patients.

\section{Competing interests}

The authors declare that they have no competing interests.

\section{References}

1. Döhner H, Weisdorf DJ and Bloomfield CD: Acute Myeloid Leukemia. N Engl J Med 373: 1136-1152, 2015.

2. Lagunas-Rangel FA, Chávez-Valencia V, Gómez-Guijosa MA and Cortes-Penagos C: Acute Myeloid Leukemia-Genetic Alterations and Their Clinical Prognosis. Int J Hematol Oncol Stem Cell Res 11: 328-339, 2017.

3. Sun Y, Chen BR and Deshpande A: Epigenetic Regulators in the Development, Maintenance, and Therapeutic Targeting of Acute Myeloid Leukemia. Front Oncol 8: 41, 2018.

4. Freireich EJ, Wiernik PH and Steensma DP: The leukemias: A half-century of discovery. J Clin Oncol 32: 3463-3469, 2014.

5. Ommen HB: Monitoring minimal residual disease in acute myeloid leukaemia: A review of the current evolving strategies. Ther Adv Hematol 7: 3-16, 2016.

6. Hourigan CS, Gale RP, Gormley NJ, Ossenkoppele GJ and Walter RB: Measurable residual disease testing in acute myeloid leukaemia. Leukemia 31: 1482-1490, 2017.
7. Zhou Y and Wood BL: Methods of Detection of Measurable Residual Disease in AML. Curr Hematol Malig Rep 12: 557-567, 2017.

8. Elmaagacli AH: Molecular methods used for detection of minimal residual disease following hematopoietic stem cell transplantation in myeloid disorders. Methods Mol Biol 1109: 187-207, 2014.

9. Tomlinson B and Lazarus HM: Enhancing acute myeloid leukemia therapy - monitoring response using residual disease testing as a guide to therapeutic decision-making. Expert Rev Hematol 10: 563-574, 2017.

10. Ross DM, Watkins DB, Hughes TP and Branford S: Reverse transcription with random pentadecamer primers improves the detection limit of a quantitative PCR assay for BCR-ABL transcripts in chronic myeloid leukemia: Implications for defining sensitivity in minimal residual disease. Clin Chem 54: 1568-1571, 2008.

11. Yang L, Han Y, Suarez Saiz F and Minden MD: A tumor suppressor and oncogene: The WT1 story. Leukemia 21: 868-876, 2007.

12. Toska E and Roberts SG: Mechanisms of transcriptional regulation by WT1 (Wilms' tumour 1). Biochem J 461: 15-32, 2014.

13. Morrison AA, Viney RL and Ladomery MR: The post-transcriptional roles of WT1, a multifunctional zinc-finger protein. Biochim Biophys Acta 1785: 55-62, 2008.

14. Lyu X, Xin Y, Mi R, Ding J, Wang X, Hu J, Fan R, Wei X, Song Y and Zhao RY: Overexpression of Wilms tumor 1 gene as a negative prognostic indicator in acute myeloid leukemia. PLoS One 9: e92470, 2014.

15. Frairia C, Aydin S, Audisio E, Riera L, Aliberti S, Allione B, Busca A, D'Ardia S, Dellacasa CM, Demurtas A, et al: Post-remissional and pre-transplant role of minimal residual disease detected by WT1 in acute myeloid leukemia: A retrospective cohort study. Leuk Res 61: 10-17, 2017.

16. Bergmann L, Miething C, Maurer U, Brieger J, Karakas T, Weidmann E and Hoelzer D: High levels of Wilms' tumor gene (wt1) mRNA in acute myeloid leukemias are associated with a worse long-term outcome. Blood 90: 1217-1225, 1997.

17. Miglino M, Colombo N, Pica G, Grasso R, Clavio M, Bergamaschi M, Ballerini F, Ghiso A, Ghiggi C, Mitscheunig L, et al: WT1 overexpression at diagnosis may predict favorable outcome in patients with de novo non-M3 acute myeloid leukemia. Leuk Lymphoma 52: 1961-1969, 2011.

18. Bennett JM, Catovsky D, Daniel MT, Flandrin G, Galton DA, Gralnick HR and Sultan C: Proposed revised criteria for the classification of acute myeloid leukemia. A report of the French-American-British Cooperative Group. Ann Intern Med 103: 620-625, 1985.

19. Vardiman JW, Thiele J, Arber DA, Brunning RD, Borowitz MJ, Porwit A, Harris NL, Le Beau MM, Hellström-Lindberg E, Tefferi A, et al: The 2008 revision of the World Health Organization (WHO) classification of myeloid neoplasms and acute leukemia: Rationale and important changes. Blood 114: 937-951, 2009.

20. Ayatollahi H, Sadeghian MH, Naderi M, Jafarian AH, Shams SF, Motamedirad N, Sheikhi M, Bahrami A and Shakeri S: Quantitative assessment of Wilms tumor 1 expression by real-time quantitative polymerase chain reaction in patients with acute myeloblastic leukemia. J Res Med Sci 22: 54, 2017.

21. Malagola M, Skert C, Ruggeri G, Turra A, Ribolla R, Cancelli V, Cattina F, Alghisi E, Bernardi S, Perucca S, et al: Peripheral blood WT1 expression predicts relapse in AML patients undergoing allogeneic stem cell transplantation. BioMed Res Int 2014: 123079, 2014.

22. Inoue K, Sugiyama H, Ogawa $H$, Nakagawa M, Yamagami $T$, Miwa H, Kita K, Hiraoka A, Masaoka T, Nasu K, et al: WT1 as a new prognostic factor and a new marker for the detection of minimal residual disease in acute leukemia. Blood 84: 3071-3079, 1994.

23. EN 13641:2002: Elimination or reduction of risk of infection related to in vitro diagnostic reagents. In: In vitro diagnostic medical devices Directive. EU Declaration of Conformity, 2017.

24. EP9-A2: Method Comparison and Bias Estimation Using Patient Samples: Approved Guideline. Vol 22. 2nd edition. NCCLS, Wayne, PA, 2002.

25. Zhu HH, Jiang H, Jiang B, Lu J, Jiang Q, Bao L, Zhang XH, Qin YZ and Huang XJ: Cytarabine, aclarubicin and granulocyte colony-stimulating factor regimen represents an effective and safe salvage regimen for patients with acute myeloid leukemia refractory to first course of induction chemotherapy. Leuk Lymphoma 54: 2452-2457, 2013. 
26. Gallego Hernanz MP, Torregrosa Diaz JM, Sorel N, Bobin A, Dindinaud E, Bouyer S, Desmier D, Brizard F, Leleu X, Maillard $\mathrm{N}$, et al: Long-term molecular remission in a patient with acute myeloid leukemia harboring a new NUP98-LEDGF rearrangement. Cancer Med 8: 1765-1770, 2019.

27. Li H, Xing C, Zhou B, Ye H, Feng J, Wu J and Gao S: A regulatory circuitry between miR-193a/miR-600 and WT1 enhances leukemogenesis in acute myeloid leukemia. Exp Hematol 61: 59-68.e5, 2018.

28. Válková V, Polák J, Marková M, Vítek A, Hájková H, Sálek C, Procházka $\mathrm{B}$, Cetkovský $\mathrm{P}$ and Trněný $\mathrm{M}$ : Minimal residual disease detectable by quantitative assessment of WT1 gene before allogeneic stem cell transplantation in patients in first remission of acute myeloid leukemia has an impact on their future prognosis. Clin Transplant 27: E21-E29, 2013.

29. Gray JX, McMillen L, Mollee P, Paul S, Lane S, Bird R, Gill D, Saal R and Marlton P: WT1 expression as a marker of minimal residual disease predicts outcome in acute myeloid leukemia when measured post-consolidation. Leuk Res 36: 453-458, 2012.

30. Mashima K, Oh I, Ikeda T, Toda Y, Ito S, Umino K, Minakata D, Nakano H, Morita K, Yamasaki R, et al: Role of Sequential Monitoring of WT1 Gene Expression in Patients With Acute Myeloid Leukemia for the Early Detection of Leukemia Relapse. Clin Lymphoma Myeloma Leuk 18: e521-e527, 2018.
31. Polák J, Hájková H, Maalaufová-Soukupová J, Marková J, Sálek C, Schwarz J and Haškovec C: Estimation of molecular upper remission limit for monitoring minimal residual disease in peripheral blood of acute myeloid leukemia patients by WT1 expression. Exp Ther Med 3: 129-133, 2012.

32. Hao Y, Cheng Y, Wu Q, Zhang A, Jiang X and Xu X: Combined usage of Wilms' tumor gene quantitative analysis and multiparameter flow cytometry for minimal residual disease monitoring of acute myeloid leukemia patients after allogeneic hematopoietic stem cells transplantation. Exp Ther Med 15: 1403-1409, 2018.

33. Duléry R, Nibourel O, Gauthier J, Elsermans V, Behal H, Coiteux V, Magro L, Renneville A, Marceau A, Boyer T, et al: Impact of Wilms' tumor 1 expression on outcome of patients undergoing allogeneic stem cell transplantation for AML. Bone Marrow Transplant 52: 539-543, 2017.

34. Candoni A, De Marchi F, Zannier ME, Lazzarotto D, Filì C, Dubbini MV, Rabassi N, Toffoletti E, Lau BW and Fanin R: High prognostic value of pre-allogeneic stem cell transplantation minimal residual disease detection by WT1 gene expression in AML transplanted in cytologic complete remission. Leuk Res 63: 22-27, 2017. 